\title{
CLASSICAL TRANSPORT THEORY AND HARD THERMAL LOOPS IN THE QUARK-GLUON PLASMA*
}

\author{
P.F. Kelly, Q. Liu, C. Lucchesi and C. Manuel \\ Center for Theoretical Physics, Laboratory for Nuclear Science, and Department of Physics \\ Massachusetts Institute of Technology, Cambridge, Massachusetts 02139
}

\begin{abstract}
Classical transport theory for colored particles is investigated and employed to derive the hard thermal loops of QCD. A formal construction of phasespace for color degrees of freedom is presented. The gauge invariance of the non-Abelian Vlasov equations is verified and used as a guiding principle in our approximation scheme. We then derive the generating functional of hard thermal loops from a constraint satisfied at leading-order by the color current. This derivation is more direct than alternative ones based on perturbative quantum field theory, and shows that hard thermal effects in hot QCD are essentially classical. As an illustration, we analyze color polarization in the QCD plasma.
\end{abstract}

Submitted to: Phys. Rev. D

PACS No: 12.38.Mh, 51.10.+y, 11.10.Wx, 11.15.Kc

CTP\# 2320 - hep-ph/9406285

June 1994

\footnotetext{
*This work is supported in part by funds provided by the U.S. Department of Energy (D.O.E.) under cooperative agreement \# DE-FC02-94ER40818. P.F.K. is supported by the Natural Sciences and Engineering Research Council of Canada. C.L. is supported by the Swiss National Science Foundation. C.M. is supported by the Ministerio de Educación y Ciencia, Spain.

Internet addresses: kelly@pierre.mit.edu (P.F.K.), liu@pierre.mit.edu (Q.L.), lucchesi@pierre.mit.edu (C.L.), cristina@pierre.mit.edu (C.M.).
} 


\section{INTRODUCTION}

Following a recent Letter [1], we present an expanded and self-contained account of the derivation of the hard thermal loops (HTLs) of QCD from classical transport theory. In addition, we justify the use of the ad hoc phase-space integration measure for classical colored particles. This justification is based on the phase-space symplectic structure, and relates directly the (dependent) color charges to a set of (independent) Darboux variables. We also discuss formally the gauge invariance properties of the system of coupled non-Abelian Vlasov equations, and exploit the gauge principle to justify the approximation scheme we use. In order to show how physical information can be extracted, we analyze color polarization of the quark-gluon plasma in a plane-wave Ansatz.

We start by reviewing the work relevant to hard thermal loops in QCD. The motivation that led to their discovery was that physical quantities (such as damping rates) in hot QCD were gauge-dependent when computed using the usual loop expansion [0]. The solution to this puzzle was first proposed by Pisarski [3]. Subsequent development was carried out by Braaten and Pisarski [4], and by Frenkel and Taylor [5]. These authors realized that, in the diagrammatic approach to high-temperature QCD, a resummation procedure is necessary in order to take into account consistently all contributions at leading-order in the coupling constant. Such contributions were found to arise only from one-loop diagrams with "soft" external and "hard" internal momenta. "Soft" denotes a scale $\sim g T$ and "hard" refers to one $\sim T$, where $g \ll 1$ is the coupling constant, and $T$ denotes the plasma temperature. Such diagrams were called "hard thermal loops" in 3,4, The HTL approach was successful in providing gauge-invariant results for physical quantities. Identifying the momentum scales that are relevant to the study of a hot quark-gluon plasma, as was done in [3, 4], was an essential step for all further developments on HTLs.

An effective action for HTLs was given by Taylor and Wong [6] who, after imposing gauge invariance, solved the resulting condition on the generating functional. Efraty and Nair [7] have identified this gauge invariance condition with the equation of motion for 
the topological Chern-Simons theory at zero temperature, thereby providing a non-thermal framework for studying hard thermal physics. Along the same line of research, the eikonal for a Chern-Simons theory has been used by Jackiw and Nair [8] to obtain a non-Abelian generalization of the Kubo formula, which governs, through the current induced by HTLs, the response of a hot quark-gluon plasma.

Another description of hard thermal loops in QCD has been proposed by Blaizot and Iancu [9]. It is based on a truncation of the Schwinger-Dyson hierarchy and yields quantum kinetic equations for the QCD induced color current. These kinetic equations, as well as the generating functional for HTLs, were obtained in [9] by performing a consistent expansion in the coupling constant, which amounts to taking into account the coupling constant dependence carried by the space-time derivatives. This dependence is extracted by going to a coordinate system which separates long-wavelength, collective excitations carrying soft momenta from the typical hard energies of plasma particles.

Alternatively, Jackiw, Liu and Lucchesi [11] have shown how HTLs can be derived from the Cornwall-Jackiw-Tomboulis composite effective action [10] by requiring its stationarity, and by using the approximation scheme developed in [9].

The resummation prescription of Braaten-Pisarski and Frenkel-Taylor, as well as the consistent expansion in the coupling constant developed by Blaizot-Iancu, although remarkably insightful, are technically very involved and necessitate lengthy computations. Furthermore, they are puzzling with respect to the very nature of hard thermal loops. One wonders if a quantum field theoretical description of hard thermal loops (involving gauge-fixing and ghost fields) is required. Indeed, we are faced with the following situation: in the resummation approach, HTLs emerge from loop diagrams, and in Blaizot and Iancu's work, they arise from the Schwinger-Dyson equations. However, hard thermal effects are UV-finite since they are due exclusively to thermal fluctuations. One might therefore be able to describe such effects within a classical, more transparent, context.

This motivated us to develop a classical formalism [1] for hard thermal loops in QCD, the natural starting point being the classical transport theory of plasmas (see for instance [12]). 
Our effort was encouraged by the fact that for an Abelian plasma of electrons and ions, the dielectric tensor computed [13] from classical transport theory is the same as that extracted from the hard thermal corrections to the vacuum polarization tensor [4, [, 8 , 8 . Moreover, the same situation is encountered for non-Abelian plasmas [14,15].

The classical transport theory for non-Abelian plasmas has been established by Elze and Heinz [14], before hard thermal effects were an issue. The HTLs of QCD were not uncovered in these early works, mainly due to the lack of a motivation to do so and because the transport equations had been linearized, thereby neglecting non-Abelian contributions. There has not been, to the best of our knowledge, any attempt to derive the complete set of HTLs for QCD from classical transport theory. The aim of the present paper is to give a detailed account of this derivation, the results of which have already appeared recently in a Letter [目].

In our approach, the generating functional of HTLs (with an arbitrary number of soft external bosonic legs) arises as a leading-order effect in the coupling constant. We start by reviewing the Wong equations for classical colored particles. Following [14, these are substituted into the transport equation, which governs the time evolution of the one-particle distribution function, thereby yielding the so-called Boltzmann equation. The latter, augmented with the Yang-Mills equation relating the field strength to the color current, form a consistent set of coupled, gauge-invariant differential equations known as non-Abelian Vlasov equations.

Expanding the distribution function in powers of the coupling constant and considering the lowest-order effects, we obtain a constraint on the color current. The latter constraint is equivalent to the condition found in [11], and previously in [9], on the induced current. The constraint on the color current leads to the generating functional of hard thermal loops.

This work is stuctured as follows. Section \1] describes classical transport theory for the quark-gluon plasma. The latter is reviewed in Subsection ПА. In Subsection ПI, we discuss and justify the ad hoc phase-space integration measure, using Darboux variables. Subsection $\amalg \mathrm{IIO}$ presents an analysis of the gauge invariance of the system of non-Abelian 
Vlasov equations. Section III contains the derivation of the hard thermal loops of QCD. As a consequence of constraints satisfied by the induced current (which are derived in Subsection [IIA), we obtain the generating functional of hard thermal loops (Subsection [IIB). In Section IV, we compute the polarization tensor from classical transport theory (at leadingorder in $g$ ) and extract the expression for Landau damping. The consistency of our result with previous ones is discussed. Section $\square$ states our conclusions. In particular, we discuss there the validity of our approximations. In Appendix A, we check the validity of the Boltzmann equation. Appendix B presents a proof of the covariant conservation of the color current.

\section{CLASSICAL TRANSPORT THEORY FOR A NON-ABELIAN PLASMA}

\section{A. Classical motion and non-Abelian Vlasov equations}

The classical transport theory for the QCD plasma was developed in [14, which we follow in this subsection. Consider a particle bearing a non-Abelian $S U(N)$ color charge $Q^{a}, a=1, \ldots, N^{2}-1$, traversing a worldline $x^{\alpha}(\tau)$, where $\tau$ denotes the proper time. The dynamical effects of the spin of the particles shall be ignored, as they are typically small. The Wong equations [16] describe the dynamical evolution of the variables $x^{\mu}, p^{\mu}$ and $Q^{a}$ :

$$
\begin{aligned}
& m \frac{d x^{\mu}}{d \tau}=p^{\mu}, \\
& m \frac{d p^{\mu}}{d \tau}=g Q^{a} F_{a}^{\mu \nu} p_{\nu}, \\
& m \frac{d Q^{a}}{d \tau}=-g f^{a b c} p^{\mu} A_{\mu}^{b} Q^{c} .
\end{aligned}
$$

\footnotetext{
${ }^{1}$ Note that we are using the kinetic momentum, rather than the canonical one. A formulation in terms of canonical variables would be equivalent [12].
} 
The $f^{a b c}$ are the structure constants of the group, $F_{a}^{\mu \nu}$ denotes the field strength, $g$ is the coupling constant, and we set $c=\hbar=k_{B}=1$ henceforth. Equation (2.1b) is the nonAbelian generalization of the Lorentz force law, and (2.10) describes the precession in color space of the charge in an external color field $A_{\mu}^{a}$. It is noteworthy that the color charge $Q^{a}$ is itself subject to dynamical evolution, a feature which distinguishes the non-Abelian theory from electromagnetism.

The usual $(x, p)$ phase-space is now enlarged to $(x, p, Q)$ by including into it color degrees of freedom for colored particles. Physical constraints are enforced by inserting deltafunctions in the phase-space volume element $d x d P d Q$. The momentum measure

$$
d P=\frac{d^{4} p}{(2 \pi)^{3}} 2 \theta\left(p_{0}\right) \delta\left(p^{2}-m^{2}\right)
$$

guarantees positivity of the energy and on-shell evolution. The color charge measure enforces the conservation of the group invariants, e.g., for $S U(3)$,

$$
d Q=d^{8} Q \delta\left(Q_{a} Q^{a}-q_{2}\right) \delta\left(d_{a b c} Q^{a} Q^{b} Q^{c}-q_{3}\right),
$$

where the constants $q_{2}$ and $q_{3}$ fix the values of the Casimirs and $d_{a b c}$ are the totally symmetric group constants. The color charges which now span the phase-space are dependent variables. These can be formally related to a set of independent phase-space Darboux variables. This derivation is presented in Subsection $\amalg \mathrm{IB}$ below.

The one-particle distribution function $f(x, p, Q)$ denotes the probability for finding the particle in the state $(x, p, Q)$. It evolves in time via a transport equation,

$$
m \frac{d f(x, p, Q)}{d \tau}=C[f](x, p, Q),
$$

where $C[f](x, p, Q)$ denotes the collision integral, which we henceforth set to zero. Using the equations of motion (2.1), (2.4) becomes, in the collisionless case, the Boltzmann equation:

$$
p^{\mu}\left[\frac{\partial}{\partial x^{\mu}}-g Q_{a} F_{\mu \nu}^{a} \frac{\partial}{\partial p_{\nu}}-g f_{a b c} A_{\mu}^{b} Q^{c} \frac{\partial}{\partial Q_{a}}\right] f(x, p, Q)=0 .
$$

In Appendix A, an explicit microscopic distribution function is presented and used to check the validity of (2.5). 
A complete, self-consistent set of non-Abelian Vlasov equations for the distribution function and the mean color field is obtained by augmenting the Boltzmann equation with the Yang-Mills equations:

$$
\left[D_{\nu} F^{\nu \mu}\right]^{a}(x)=J^{\mu a}(x)
$$

The covariant derivative is defined as $D_{\mu}^{a c}=\partial_{\mu} \delta^{a c}+g f^{a b c} A_{\mu}^{b}$. The total color current $J^{\mu a}(x)$ is given by the sum of all contributions from particle species and helicities,

$$
J^{\mu a}(x)=\sum_{\text {species }} \sum_{\text {helicities }} j^{\mu a}(x)
$$

Each $j^{\mu a}(x)$ (species and spin indices are implicit) is computed from the corresponding distribution function as

$$
j^{\mu a}(x)=g \int d P d Q p^{\mu} Q^{a} f(x, p, Q)
$$

and it is covariantly conserved,

$$
\left(D_{\mu} j^{\mu}\right)^{a}(x)=0
$$

as can be checked by using the Boltzmann equation (a detailed proof is presented in Appendix B]). For later convenience, we define the total and individual current momentumdensities:

$$
J^{\mu a}(x, p)=\sum_{\text {species }} \sum_{\text {helicities }} j^{\mu a}(x, p), \quad j^{\mu a}(x, p)=g \int d Q p^{\mu} Q^{a} f(x, p, Q)
$$

Note that a solution to the set of Vlasov equations (2.5)-(2.6) is specified by giving the forms for the gauge potential $A_{\mu}(x)$ and for the distribution function $f(x, p, Q)$.

\section{B. Phase-space for colored particles}

In order to carry out the transport theory analysis for classical colored particles, it has

been necessary to extend phase-space by the addition of the color charges. In (2.3), the charges are constrained to remain within the group manifold by means of delta-functions 
which fix the values of the (representation-dependent) group Casimirs. In fact, at an operational level, this is the approach adopted in the rest of the present paper. In this subsection, we formally justify this approach by analysis of the symplectic structure of the group manifold [19, 18]. We work out explicitly the $S U(2)$ and $S U(3)$ cases.

The group $S U(2)$, is generated by three charges, $\left(Q_{1}, Q_{2}, Q_{3}\right)$, and has one Casimir, $Q^{a} Q_{a}$. The structure constants are $f_{a b c}=\epsilon_{a b c}$, while $d_{a b c}=0$. From the point of view adopted throughout the rest of this paper the phase-space color measure is

$$
d Q=d Q_{1} d Q_{2} d Q_{3} \delta\left(Q^{a} Q_{a}-q_{2}\right)
$$

where $q_{2}$ denotes the value of the quadratic Casimir.

New coordinates $(\phi, \pi, J)$ may be introduced by the following transformation [18]:

$$
Q_{1}=\cos \phi \sqrt{J^{2}-\pi^{2}}, \quad Q_{2}=\sin \phi \sqrt{J^{2}-\pi^{2}}, \quad Q_{3}=\pi
$$

Note that $\pi$ is bounded, $-J \leq \pi \leq J$. That the group manifold has spherical geometry is readily apparent if one chooses $\pi=J \cos \theta$. The variables $\phi$ and $\pi$ form a canonically conjugate pair; the Poisson bracket may be formed in the conventional manner:

$$
\{A, B\}_{\mathrm{PB}} \equiv \frac{\partial A}{\partial \phi} \frac{\partial B}{\partial \pi}-\frac{\partial A}{\partial \pi} \frac{\partial B}{\partial \phi} .
$$

It is easily verified that the charges as given by (2.12) form a representation of $S U(2)$ under the Poisson bracket, i.e.,

$$
\left\{Q_{a}, Q_{b}\right\}_{\mathrm{PB}}=\epsilon_{a b c} Q_{c}
$$

The above Poisson bracket structure allows one to identify $\phi$ and $\pi$ as Darboux variables (see for instance [19]). The Jacobian of the transformation from $\left(Q_{1}, Q_{2}, Q_{3}\right)$ to $(\phi, \pi, J)$ takes the value

$$
\left|\frac{\partial\left(Q_{1}, Q_{2}, Q_{3}\right)}{\partial(\phi, \pi, J)}\right|=J .
$$

Performing the change of variables (2.12) in (2.11) and substituting the value of the quadratic Casimir, $Q^{a} Q_{a}=J^{2}$, the color measure reads 


$$
d Q=d \phi d \pi d J J \delta\left(J^{2}-q_{2}\right),
$$

which, upon integration over the constrained variable $J$, is just the proper, canonical volume element $d \phi d \pi$, up to an irrelevant constant.

The group $S U(3)$ has eight charges, $\left(Q_{1}, \ldots, Q_{8}\right)$ and two conserved quantities, the quadratic and the cubic Casimirs, $Q^{a} Q_{a}$ and $d_{a b c} Q^{a} Q^{b} Q^{c}$, respectively. The phase-space color measure is quoted above in (2.3).

As in the $S U(2)$ case, new coordinates $\left(\phi_{1}, \phi_{2}, \phi_{3}, \pi_{1}, \pi_{2}, \pi_{3}, J_{1}, J_{2}\right)$ may be introduced by means of the following transformations [18]:

$$
\begin{aligned}
& Q_{1}=\cos \phi_{1} \pi_{+} \pi_{-}, \quad Q_{2}=\sin \phi_{1} \pi_{+} \pi_{-}, \\
& Q_{3}=\pi_{1} \\
& Q_{4}=C_{++} \pi_{+} A+C_{+-} \pi_{-} B, \quad Q_{5}=S_{++} \pi_{+} A+S_{+-} \pi_{-} B, \\
& Q_{6}=C_{-+} \pi_{-} A-C_{--} \pi_{+} B, \quad Q_{7}=S_{-+} \pi_{-} A-S_{--} \pi_{+} B, \\
& Q_{8}=\pi_{2},
\end{aligned}
$$

in which we have used the definitions:

$$
\begin{aligned}
\pi_{+} & =\sqrt{\pi_{3}+\pi_{1}}, & \pi_{-} & =\sqrt{\pi_{3}-\pi_{1}}, \\
C_{ \pm \pm} & =\cos \left[\frac{1}{2}\left( \pm \phi_{1}+\sqrt{3} \phi_{2} \pm \phi_{3}\right)\right], & S_{ \pm \pm} & =\sin \left[\frac{1}{2}\left( \pm \phi_{1}+\sqrt{3} \phi_{2} \pm \phi_{3}\right)\right],
\end{aligned}
$$

and $A, B$ are given by

$$
\begin{aligned}
& A=\frac{1}{2 \pi_{3}} \sqrt{\left(\frac{J_{1}-J_{2}}{3}+\pi_{3}+\frac{\pi_{2}}{\sqrt{3}}\right)\left(\frac{J_{1}+2 J_{2}}{3}+\pi_{3}+\frac{\pi_{2}}{\sqrt{3}}\right)\left(\frac{2 J_{1}+J_{2}}{3}-\pi_{3}-\frac{\pi_{2}}{\sqrt{3}}\right)}, \\
& B=\frac{1}{2 \pi_{3}} \sqrt{\left(\frac{J_{2}-J_{1}}{3}+\pi_{3}-\frac{\pi_{2}}{\sqrt{3}}\right)\left(\frac{J_{1}+2 J_{2}}{3}-\pi_{3}+\frac{\pi_{2}}{\sqrt{3}}\right)\left(\frac{2 J_{1}+J_{2}}{3}+\pi_{3}-\frac{\pi_{2}}{\sqrt{3}}\right)} .
\end{aligned}
$$

Note that in this representation, the set $\left(Q_{1}, Q_{2}, Q_{3}\right)$ forms an $S U(2)$ subgroup with quadratic Casimir $Q_{1}^{2}+Q_{2}^{2}+Q_{3}^{2}=\pi_{3}^{2}$. It can be verified that the expressions above for $Q_{1}, \ldots, Q_{8}$ form a representation of the group $S U(3)$ :

$$
\left\{Q_{a}, Q_{b}\right\}_{\mathrm{PB}}=f_{a b c} Q_{c},
$$

under the Poisson bracket 


$$
\{A, B\}_{\mathrm{PB}} \equiv \sum_{i=1}^{3}\left(\frac{\partial A}{\partial \phi_{i}} \frac{\partial B}{\partial \pi_{i}}-\frac{\partial A}{\partial \pi_{i}} \frac{\partial B}{\partial \phi_{i}}\right),
$$

where the canonical pairs are $\left\{\phi_{i}, \pi_{i}\right\}_{i=1,2,3}$.

As is implicit in the above, the two Casimirs depend only on $J_{1}$ and $J_{2}$. They can be computed, using the values given in the table below, as:

$$
\begin{aligned}
Q^{a} Q_{a} & =\frac{1}{3}\left(J_{1}^{2}+J_{1} J_{2}+J_{2}^{2}\right), \\
d_{a b c} Q^{a} Q^{b} Q^{c} & =\frac{1}{18}\left(J_{1}-J_{2}\right)\left(J_{1}+2 J_{2}\right)\left(2 J_{1}+J_{2}\right) .
\end{aligned}
$$

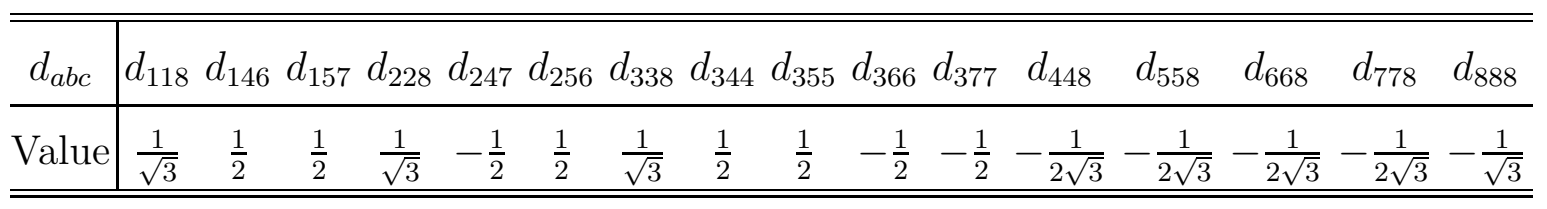

Table: Values of the (non-zero) $S U(3)$ totally symmetric constants.

The phase-space color measure for $S U(3)$, given in (2.3), may be transformed to the new coordinates through use of (2.21) and evaluation of the Jacobian

$$
\left|\frac{\partial\left(Q_{1}, Q_{2}, \ldots, Q_{8}\right)}{\partial\left(\phi_{1}, \phi_{2}, \phi_{3}, \pi_{1}, \pi_{2}, \pi_{3}, J_{1}, J_{2}\right)}\right|=\frac{\sqrt{3}}{48} J_{1} J_{2}\left(J_{1}+J_{2}\right) .
$$

The measure reads:

$$
\begin{aligned}
d Q=d \phi_{1} d \phi_{2} d \phi_{3} d \pi_{1} d \pi_{2} d \pi_{3} d J_{1} d J_{2} & \frac{\sqrt{3}}{48} J_{1} J_{2}\left(J_{1}+J_{2}\right) \delta\left(\frac{1}{3}\left(J_{1}^{2}+J_{1} J_{2}+J_{2}^{2}\right)-q_{2}\right) \times \\
& \delta\left(\frac{1}{18}\left(J_{1}-J_{2}\right)\left(J_{1}+2 J_{2}\right)\left(2 J_{1}+J_{2}\right)-q_{3}\right)
\end{aligned}
$$

Since the two Casimirs are linearly independent, the delta-functions uniquely fix both $J_{1}$ and $J_{2}$ to be representation-dependent constants. Upon integrating over $J_{1}$ and $J_{2}$, (2.23) reduces to a constant times the proper canonical volume element $\prod_{i=1}^{3} d \phi_{i} d \pi_{i}$.

The construction of the canonical phase-space measure for the general $S U(N)$ case is a departure from our purposes and will not be undertaken here. Nevertheless, based on the examples we treated explicitly, it is apparent that no difficulties will arise for $N>3$ [19].

In principle, the classical transport theory analysis should be carried out using canonical, independent integration variables and the phase-space volume element should be taken to 
be the proper canonical volume element. In this subsection, we have shown the equivalence of the ad hoc phase-space color measure and the proper canonical volume element. Hence, the use of the color charges as phase-space coordinates is justified.

\section{Gauge invariance of the non-Abelian Vlasov equations}

Before addressing the question of the gauge invariance of the system of Vlasov equations, we consider the Wong equations (2.1). These are invariant under the finite gauge transformations:

$$
\begin{aligned}
\bar{x}^{\mu} & =x^{\mu}, \\
\bar{p}^{\mu} & =p^{\mu}, \\
\bar{Q} & =U Q U^{-1}, \\
\bar{A}_{\mu} & =U A_{\mu} U^{-1}-\frac{1}{g} U \frac{\partial}{\partial x_{\mu}} U^{-1},
\end{aligned}
$$

where $U(x)=\exp \left[-g \varepsilon^{a}(x) t^{a}\right]$ is a group element.

Accordingly, the derivatives appearing in the Boltzmann equation (2.5) transform as:

$$
\begin{aligned}
\frac{\partial}{\partial x^{\mu}} & =\frac{\partial}{\partial \bar{x}^{\mu}}-2 \operatorname{Tr}\left(\left[\left(\frac{\partial}{\partial \bar{x}^{\mu}} U\right) U^{-1}, \bar{Q}\right] \frac{\partial}{\partial \bar{Q}}\right) \\
\frac{\partial}{\partial p^{\mu}} & =\frac{\partial}{\partial \bar{p}^{\mu}} \\
\frac{\partial}{\partial Q} & =U^{-1} \frac{\partial}{\partial \bar{Q}} U .
\end{aligned}
$$

Consequently, the Boltzmann equation (rewritten here in terms of traces):

$$
\left[p^{\mu} \frac{\partial}{\partial x^{\mu}}-2 g p^{\mu} \operatorname{Tr}\left(Q F_{\mu \nu}\right) \frac{\partial}{\partial p_{\nu}}+2 g p^{\mu} \operatorname{Tr}\left(\left[A_{\mu}, Q\right] \frac{\partial}{\partial Q}\right)\right] f(x, p, Q)=0
$$

\footnotetext{
${ }^{2}$ We use here matrix notation, e.g. $Q=Q_{a} t^{a}, \frac{\partial}{\partial Q}=\frac{\partial}{\partial Q^{a}} t^{a}$, where the generators are represented by antihermitian matrices $t^{a}$ in the fundamental representation, $\left[t^{a}, t^{b}\right]=f^{a b c} t^{c}$, and they are normalized as $\operatorname{Tr}\left(t^{a} t^{b}\right)=-\frac{1}{2} \delta^{a b}$.
} 
becomes, in the new coordinates:

$$
\begin{aligned}
& {\left[\bar{p}^{\mu} \frac{\partial}{\partial \bar{x}^{\mu}}-2 g \bar{p}^{\mu} \operatorname{Tr}\left(\left[\left(\frac{\partial}{\partial \bar{x}^{\mu}} U\right) U^{-1}, \bar{Q}\right] \frac{\partial}{\partial \bar{Q}}\right)+2 g \bar{p}^{\mu} \operatorname{Tr}\left(\bar{Q} \bar{F}_{\mu \nu}\right) \frac{\partial}{\partial \bar{p}_{\nu}}\right.} \\
& \left.+2 g \bar{p}^{\mu} \operatorname{Tr}\left(\left[\bar{A}_{\mu}+\frac{1}{g}\left(\frac{\partial}{\partial \bar{x}^{\mu}} U\right) U^{-1}, \bar{Q}\right] \frac{\partial}{\partial \bar{Q}}\right)\right] \bar{f}(\bar{x}, \bar{p}, \bar{Q})=0,
\end{aligned}
$$

where we have defined

$$
\bar{f}(\bar{x}, \bar{p}, \bar{Q})=f(x(\bar{x}, \bar{p}, \bar{Q}), p(\bar{x}, \bar{p}, \bar{Q}), Q(\bar{x}, \bar{p}, \bar{Q})) .
$$

Simplifying (2.27), we obtain:

$$
\left[\bar{p}^{\mu} \frac{\partial}{\partial \bar{x}^{\mu}}+2 g \bar{p}^{\mu} \operatorname{Tr}\left(\bar{Q} \bar{F}_{\mu \nu}\right) \frac{\partial}{\partial \bar{p}_{\nu}}+2 g \bar{p}^{\mu} \operatorname{Tr}\left(\left[\bar{A}_{\mu}, \bar{Q}\right] \frac{\partial}{\partial \bar{Q}}\right)\right] \bar{f}(\bar{x}, \bar{p}, \bar{Q})=0 .
$$

This proves that the Boltzmann equation is invariant under gauge transformations. On the other hand, the Yang-Mills equation (2.6) is gauge-covariant. Indeed, the color current (2.8) transforms under (2.24) as a gauge covariant vector: $j^{\mu}(x) \rightarrow \bar{j}^{\mu}(\bar{x})=$ $\int d \bar{P} d \bar{Q} \bar{p}^{\mu} \bar{Q} \bar{f}(\bar{x}, \bar{p}, \bar{Q})$. Due to the gauge-invariance of the phase-space measure, to the transformation property of $f(2.28)$, and to (2.24), $\bar{j}^{\mu}(x)$ may be rewritten as:

$$
\bar{j}^{\mu}(\bar{x})=\int d P d Q p^{\mu} U Q U^{-1} f(x, p, Q)=U j^{\mu}(x) U^{-1} .
$$

Hence, the system of non-Abelian Vlasov equations is gauge-covariant, with the distribution function $f(x, p, Q)$ transforming as a scalar. Note that the gauge symmetry also implies that the gauge transform $\left\{\bar{A}_{\mu}(x), \bar{f}(x, p, Q)\right\}$ of a set of solutions $\left\{A_{\mu}(x), f(x, p, Q)\right\}$ to the Vlasov equations:

$$
\begin{aligned}
& \bar{A}_{\mu}(x)=U A_{\mu}(x) U^{-1}-\frac{1}{g} U \frac{\partial}{\partial x^{\mu}} U^{-1} \\
& \bar{f}(x, p, Q)=f\left(x, p, U Q U^{-1}\right)
\end{aligned}
$$

is still a solution.

\section{EMERGENCE OF HARD THERMAL LOOPS}




\section{A. Constraint on the color current}

Classical transport theory is now employed to study soft excitations in a hot, colorneutral quark-gluon plasma. In the high-temperature limit, the masses of the particles can be neglected and shall henceforth be assumed to vanish. The wavelength of a soft excitation is of order $\frac{1}{g|A|}$ and the coupling constant $g$ is assumed to be small. We then expand the distribution function $f(x, p, Q)$ in powers of $g$ :

$$
f=f^{(0)}+g f^{(1)}+g^{2} f^{(2)}+\ldots,
$$

where $f^{(0)}$ is the equilibrium distribution function in the absence of a net color field, and is given by:

$$
f^{(0)}\left(p_{0}\right)=C n_{B, F}\left(p_{0}\right)
$$

Here $C$ is a normalization constant and $n_{B, F}\left(p_{0}\right)=1 /\left(e^{\beta\left|p_{0}\right|} \mp 1\right)$ is the bosonic, resp. fermionic, probability distribution.

At leading-order in $g$, the color current 2.10$)$ is

$$
j^{\mu a}(x, p)=g^{2} \int d Q p^{\mu} Q^{a} f^{(1)}(x, p, Q)
$$

while the Boltzmann equation (2.5) reduces to

$$
p^{\mu}\left(\frac{\partial}{\partial x^{\mu}}-g f^{a b c} A_{\mu}^{b} Q_{c} \frac{\partial}{\partial Q^{a}}\right) f^{(1)}(x, p, Q)=p^{\mu} Q_{a} F_{\mu \nu}^{a} \frac{\partial}{\partial p_{\nu}} f^{(0)}\left(p_{0}\right) .
$$

Due to the softness of the excitation, the $\frac{\partial}{\partial x^{\mu}}$ in the above equation is of order $g|A|$, so we are taking into account consistently all contributions of order $g$. The approximation we use guarantees that the non-Abelian gauge symmetry of the exact Boltzmann equation (2.5) is preserved in the approximate equation (3.4). As a consequence $f^{(0)}$ and $f^{(1)}$, like $f$, transform separately as gauge-invariant scalars. Other approximations, which have been

carried out in the past [14, have discarded the non-Abelian contributions, thereby breaking the non-Abelian gauge symmetry of the Boltzmann equation.

The equations (3.3) and (3.4) yield the following constraint on the color current: 


$$
\left[p \cdot D j^{\mu}(x, p)\right]^{a}=g^{2} p^{\mu} p^{\nu} F_{\nu \rho}^{b} \frac{\partial}{\partial p_{\rho}}\left(\int d Q Q^{a} Q_{b} f^{(0)}\left(p_{0}\right)\right),
$$

where, from color symmetry, we have $\int d Q Q^{a} Q_{b} f^{(0)}\left(p_{0}\right)=C_{B, F} n_{B, F}\left(p_{0}\right) \delta_{b}^{a}$ with $C_{B}=$ $N, C_{F}=\frac{1}{2}$ for gluons, resp. fermions. Thus, upon summation over all species ( $N_{F}$ quarks, $N_{F}$ antiquarks and one [( $\left.N^{2}-1\right)$-plet] gluon) and helicities (2 for quarks-antiquarks and for the massless gluon), (3.5) yields,

$$
\left[p \cdot D J^{\mu}(x, p)\right]^{a}=2 g^{2} p^{\mu} p^{\nu} F_{\nu 0}^{a} \frac{d}{d p_{0}}\left[N n_{B}\left(p_{0}\right)+N_{F} n_{F}\left(p_{0}\right)\right] .
$$

Similar results have been obtained in [9.11], in a quantum field theoretic setting.

\section{B. Derivation of hard thermal loops}

Subsequent steps which lead to the generating functional of HTLs have been described in [11], the results of which were used straightforwardly in [1], for the sake of brevity. Here, we present a simpler derivation of HTLs by exploiting fully the structure of the momentum integration measure (2.2).

We first integrate equation (3.6) over $|\mathbf{p}|$ and $p_{0}$ using the massless limit of the momentum measure $d P(2.2)$. Therefore, the (massless) mass-shell constraint enforces $|\mathbf{p}|=p_{0}$, and we

thus introduce the unit vector $\hat{\mathbf{p}} \equiv \mathbf{p} /|\mathbf{p}|$. Introducing also $v \equiv(1, \hat{\mathbf{p}})$, the integration of (3.6) yields (group indices are henceforth omitted):

$$
v \cdot D \mathcal{J}^{\mu}(x, v)=-2 \pi^{2} m_{D}^{2} v^{\mu} v^{\rho} F_{\rho 0}(x),
$$

where $m_{D}$ is the Debye screening mass

$$
m_{D}=g T \sqrt{\frac{N+N_{F} / 2}{3}},
$$

and we have defined

$$
\mathcal{J}^{\mu}(x, v)=\int|\mathbf{p}|^{2} d|\mathbf{p}| d p_{0} 2 \theta\left(p_{0}\right) \delta\left(p^{2}\right) J^{\mu}(x, p) .
$$


Notice (for later use) that, using $\int d P=\int \frac{d \Omega}{(2 \pi)^{3}}|\mathbf{p}|^{2} d|\mathbf{p}| d p_{0} 2 \theta\left(p_{0}\right) \delta\left(p^{2}\right)$, where $d \Omega$ denotes integration over all angular directions of the unit vector $\hat{\mathbf{p}}$, we can rewrite the expression $J^{\mu}(x)=\int d P J^{\mu}(x, p)$ for the color current as

$$
J^{\mu}(x)=\int \frac{d \Omega}{(2 \pi)^{3}} \mathcal{J}^{\mu}(x, v)
$$

After decomposing $\mathcal{J}^{\mu}(x, v)$ as

$$
\mathcal{J}^{\mu}(x, v)=\tilde{\mathcal{J}}^{\mu}(x, v)-2 \pi^{2} m_{D}^{2} v^{\mu} A_{0}(x)
$$

we get as our final condition on the color current:

$$
v \cdot D \tilde{\mathcal{J}}^{\mu}(x, v)=2 \pi^{2} m_{D}^{2} v^{\mu} \frac{\partial}{\partial x^{0}}(v \cdot A(x))
$$

It has been shown that solutions to (3.12) can be obtained from a functional $W(A, v)$ as [6]

$$
\tilde{\mathcal{J}}^{\mu}(x, v)=\frac{\delta W(A, v)}{\delta A_{\mu}(x)} .
$$

Equation (3.12) then implies that $W(A, v)$ depends only on $A_{+} \equiv v \cdot A$, i.e. $W(A, v)=$ $W\left(A_{+}\right)$, and $\tilde{\mathcal{J}}^{\mu}=\frac{\delta W\left(A_{+}\right)}{\delta A_{+}} v^{\mu}$. In turn, $W\left(A_{+}\right)$satisfies, as a consequence of (3.12),

$$
v \cdot D \frac{\delta W\left(A_{+}\right)}{\delta A_{+}}=2 \pi^{2} m_{D}^{2} \frac{\partial}{\partial x^{0}} A_{+} .
$$

By introducing new coordinates $\left(x_{+}, x_{-}, \mathbf{x}_{\perp}\right)$,

$$
x_{+}=\bar{v} \cdot x, \quad x_{-}=v \cdot x, \quad \mathbf{x}_{\perp}=\mathbf{x}-(\hat{\mathbf{p}} \cdot \mathbf{x}) \hat{\mathbf{p}}
$$

with $\bar{v} \equiv(1,-\hat{\mathbf{p}})$ and $\mathbf{x}_{\perp} \cdot \hat{\mathbf{p}}=0$, we can rewrite $v \cdot \frac{\partial}{\partial x}$ as $\partial_{+}$and (3.14) becomes:

$$
\partial_{+} \frac{\delta W\left(A_{+}\right)}{\delta A_{+}}+g\left[A_{+}, \frac{\delta W\left(A_{+}\right)}{\delta A_{+}}\right]=2 \pi^{2} m_{D}^{2} \frac{\partial}{\partial x^{0}} A_{+} .
$$

Now using (3.10), (3.11) and (3.13), we define an effective action $\Gamma$ that generates the color current, i.e., $J^{\mu}(x)=-\frac{\delta \Gamma[A(x)]}{\delta A_{\mu}(x)}$, where $\Gamma$ takes the form:

$$
\Gamma=\frac{m_{D}^{2}}{2} \int d^{4} x A_{0}^{a}(x) A_{0}^{a}(x)-\int \frac{d \Omega}{(2 \pi)^{3}} W\left(A_{+}\right) .
$$


This is the expression for the effective action generating hard thermal loops [4, 5], while equation (3.16) represents the condition of gauge invariance 6] for this generating functional. By solving (3.16), Taylor and Wong [6], as well as Efraty and Nair [7], have given an explicit form for the functional $W\left(A_{+}\right)$in the second term of (3.17). The first term is a mass term for $A_{0}^{a}(x)$ and describes Debye screening.

This concludes our derivation of the hard thermal loops of QCD from classical transport theory.

\section{APPLICATION: COLOR POLARIZATION}

As an application of the classical transport formalism presented above, we solve the approximate Boltzmann equation (3.4) for plane-wave excitations in a collisionless isotropic plasma of quarks and gluons. Recall that in the case of a collisionless plasma of electrons and ions the Abelian version of equation (3.4) has been solved exactly for an electromagnetic plane-wave [12,13], making it possible to study the response of an Abelian plasma to a weak field. We shall proceed analogously in the non-Abelian case. We consider a plane-wave Ansatz in which the vector gauge fields only depend on $x^{\mu}$ through the combination $x \cdot k$, where $k^{\mu}=(\omega, \mathbf{k})$ is the wave vector, i.e., $A_{\mu}^{a}(x) \equiv A_{\mu}^{a}(k \cdot x)$. With this Ansatz (which has been used in [17] to study the non-Abelian Kubo equation) the solution of (3.4) is

$$
f^{(1)}(x, p, Q)=Q_{a}\left(A_{0}^{a}(x)-\omega \frac{p \cdot A^{a}(x)}{p \cdot k}\right) \frac{d}{d p_{0}} f^{(0)}\left(p_{0}\right) .
$$

Hence, the color current is given by

$$
j_{a}^{\mu}(x)=g^{2} \int d P d Q p^{\mu} Q_{a} Q_{b}\left(A_{0}^{b}(x)-\omega \frac{p \cdot A^{b}(x)}{p \cdot k}\right) \frac{d}{d p_{0}} f^{(0)}\left(p_{0}\right) .
$$

The integration over color charges can be done by using $\int d Q Q^{a} Q_{b}=C_{B, F} \delta_{b}^{a}$ with $C_{B}=$

$N, C_{F}=\frac{1}{2}$ for gluons, resp. fermions. The integration over $p_{0}$ and $|\mathbf{p}|$ is straighforward as well. Upon summation over all species and helicities (see Section III for notations and conventions), we get the following expression for the total color current: 


$$
J_{a}^{\mu}(x)=m_{D}^{2} \int \frac{d \Omega}{4 \pi} v^{\mu}\left(\omega \frac{v \cdot A_{a}(x)}{v \cdot k}-A_{a}^{0}(x)\right) .
$$

The polarization tensor $\Pi_{a b}^{\mu \nu}$ can be computed from (4.3) by using the relation

$$
J_{a}^{\mu}(x)=\int d^{4} y \Pi_{a b}^{\mu \nu}(x-y) A_{\nu}^{b}(y)
$$

It reads:

$$
\Pi_{a b}^{\mu \nu}(x-y)=m_{D}^{2}\left(-g^{\mu 0} g^{\nu 0}+\omega I^{\mu \nu}(\omega, \mathbf{k})\right) \delta^{(4)}(x-y) \delta_{a b}
$$

where $I^{\mu \nu}$ is defined as

$$
I^{\mu \nu}(\omega, \mathbf{k})=\int \frac{d \Omega}{4 \pi} \frac{v^{\mu} v^{\nu}}{\omega-\mathbf{k} \cdot \mathbf{v}}
$$

To avoid the poles in the above integrand, we impose retarded boundary conditions, i.e., we replace $\omega$ by $\omega+i \epsilon$. Using the identity

$$
\frac{1}{z+i \epsilon}=\mathcal{P} \frac{1}{z}-i \pi \delta(z)
$$

where $\mathcal{P}$ stands for the principal value, the real and imaginary parts of the polarization tensor are

$$
\begin{aligned}
& \operatorname{Re} \Pi_{a b}^{\mu \nu}(\omega, \mathbf{k})=-\delta_{a b} m_{D}^{2}\left(-g^{\mu 0} g^{\nu 0}+\omega \mathcal{P} \int \frac{d \Omega}{4 \pi} \frac{v^{\mu} v^{\nu}}{\omega-\mathbf{k} \cdot \mathbf{v}}\right), \\
& \operatorname{Im} \Pi_{a b}^{\mu \nu}(\omega, \mathbf{k})=-\delta_{a b} m_{D}^{2} \pi \omega \int \frac{d \Omega}{4 \pi} v^{\mu} v^{\nu} \delta(\omega-\mathbf{k} \cdot \mathbf{v}) .
\end{aligned}
$$

The imaginary part of the polarization tensor (4.8b) describes Landau damping in the quark-gluon plasma. Explicitly:

$$
\begin{aligned}
& \operatorname{Im} \Pi_{a b}^{00}(\omega, \mathbf{k})=-\delta_{a b} m_{D}^{2} \pi \frac{\omega}{2|\mathbf{k}|} \theta\left(|\mathbf{k}|^{2}-\omega^{2}\right) \\
& \operatorname{Im} \Pi_{a b}^{0 i}(\omega, \mathbf{k})=-\delta_{a b} m_{D}^{2} \pi \frac{\omega^{2}}{2|\mathbf{k}|^{2}} \frac{k^{i}}{|\mathbf{k}|} \theta\left(|\mathbf{k}|^{2}-\omega^{2}\right), \\
& \operatorname{Im} \Pi_{a b}^{i j}(\omega, \mathbf{k})=-\delta_{a b} m_{D}^{2} \pi\left[\frac{\omega^{2}}{4|\mathbf{k}|^{2}}\left(\frac{|\mathbf{k}|}{\omega}-\frac{\omega}{|\mathbf{k}|}\right)\left(\delta^{i j}-\frac{k^{i} k^{j}}{|\mathbf{k}|^{2}}\right)+\frac{\omega^{3}}{2|\mathbf{k}|^{3}} \frac{k^{i} k^{j}}{|\mathbf{k}|^{2}}\right] \theta\left(|\mathbf{k}|^{2}-\omega^{2}\right) .
\end{aligned}
$$

From the above $\theta$-functions it is apparent that Landau damping only occurs for color fields with space-like wave vectors. This is also true for an Abelian plasma [12]. 
Evaluation of the real part of the polarization tensor (4.8a) yields

$$
\begin{aligned}
& \operatorname{Re} \Pi_{a b}^{00}(\omega, \mathbf{k})=\delta_{a b} \Pi_{l}(\omega, \mathbf{k}), \\
& \operatorname{Re} \Pi_{a b}^{0 i}(\omega, \mathbf{k})=\delta_{a b} \omega \frac{k^{i}}{|\mathbf{k}|^{2}} \Pi_{l}(\omega, \mathbf{k}), \\
& \operatorname{Re} \Pi_{a b}^{i j}(\omega, \mathbf{k})=\delta_{a b}\left[\left(\delta^{i j}-\frac{k^{i} k^{j}}{|\mathbf{k}|^{2}}\right) \Pi_{t}(\omega, \mathbf{k})+\frac{k^{i} k^{j}}{|\mathbf{k}|^{2}} \frac{\omega^{2}}{|\mathbf{k}|^{2}} \Pi_{l}(\omega, \mathbf{k})\right],
\end{aligned}
$$

where

$$
\begin{aligned}
& \Pi_{l}(\omega, \mathbf{k})=m_{D}^{2}\left(\frac{\omega}{2|\mathbf{k}|} \ln \left|\frac{\omega+|\mathbf{k}|}{\omega-|\mathbf{k}|}\right|-1\right), \\
& \Pi_{t}(\omega, \mathbf{k})=-m_{D}^{2} \frac{\omega^{2}}{2|\mathbf{k}|^{2}}\left[1+\frac{1}{2}\left(\frac{|\mathbf{k}|}{\omega}-\frac{\omega}{|\mathbf{k}|}\right) \ln \left|\frac{\omega+|\mathbf{k}|}{\omega-|\mathbf{k}|}\right|\right] .
\end{aligned}
$$

Equations (4.10)-(4.11) characterize Debye screening, as well as longitudinal and transverse plasma waves.

Our results for the HTLs of the polarization tensor agree with those obtained in the high temperature limit using quantum field theoretic techniques [15, 15,8,8,9, 17. We emphasize that the above results are gauge-independent, and obey the Ward identity

$$
k_{\mu} \Pi_{a b}^{\mu \nu}=0,
$$

as should be expected from the gauge invariance of our formalism.

Previous applications of classical transport theory to QCD have utilized an Abeliandominance approximation to compute the polarization tensor [14]. It is noteworthy that there one recovers the same values of the polarization tensor that we found here. The reason for this agreement is that the leading-order contribution to the color current is made linear in the gauge field by the plane-wave Ansatz [17], exactly as happens in the Abeliandominance approximation. However, the Abelian-dominance approximation cannot give a proper account of the whole set of HTLs, such as thermal corrections to $n$-point functions, $n \geq 3$.

\footnotetext{
${ }^{3}$ The connection between the retarded polarization tensor computed here and the time-ordered polarization tensor that is commonly used in quantum field theory has been studied in [8].
} 


\section{CONCLUSIONS}

In this paper, we have shown how classical transport theory can be used to derive the hard thermal loops of QCD. This formalism, we believe, is more direct and transparent than previous approaches based on perturbative quantum field theory. Indeed, hard thermal loops represent UV-finite thermal corrections to propagators and vertices. They arise from thermal scattering within a hot assembly of particles, and one may reasonably expect them to be describable in terms of classical physics.

The fact that we are modelling the high-temperature, deconfined, phase of QCD allows us to treat color classically, and enables the colored constituents of the plasma to be identified as quarks and gluons. Employing classical transport theory to study colored particles requires incorporating the color degrees of freedom into phase-space. A consistent measure must be defined over the new color coordinates. Furthermore, conservation of the group Casimirs under the dynamical evolution must be ensured. One means to accomplish these goals is to include delta-function constraints into the phase-space volume element. We have formally justified this ad hoc procedure by relating the dependent color degrees of freedom to a set of independent Darboux canonical variables, and by proving that the corresponding volume elements are equivalent.

A system of non-Abelian Vlasov equations describes transport phenomena in the QCD plasma. This system governs the evolution of both the single-particle phase-space distribution functions and the mean color fields. It would be a formidable task to solve the transport equation in the most general case, hence suitable approximations must be made.

First, we specialize to a collisionless plasma, in which there is no direct scattering between particles. This situation is not devoid of interest since collective mean-field effects can, and indeed do, arise.

Second, we employ a perturbative approximation scheme. We assume that the plasma is near equilibrium and expand the phase-space distribution function in powers of $g$, the gauge coupling constant. At the high temperature which must prevail for the formation 
of a quark-gluon plasma, the thermal energies of the particles are sufficiently large that the effects of the interactions with the gauge fields are comparatively small, and we expect perturbation theory to be valid.

Taking the high temperature limit constitutes our third approximation. In this limit, the masses of the particles can be neglected. Furthermore, the plasma is in a highly degenerate state, so that the equilibrium distribution functions are determined by the spin-statistics theorem.

We demand that gauge invariance be preserved by our perturbative expansion. In its lowest, non-trivial, order this expansion leads to the generating functional of HTLs. That gauge invariance is the appropriate guiding principle in uncovering hard thermal loops is not surprising. To apply this principle to the quantum field theoretic calculation of HTLs involves gauge fixing, ghosts, and resummation of classes of Feynman diagrams. In contrast, the route that one has to follow in order to adhere to the gauge principle is straightforward within classical physics. Therefore, the gauge invariance property of hard thermal loops is self-evident in our formalism.

Acknowledgements: We thank Professor R. Jackiw for constant encouragement and for many useful and enjoyable discussions.

\section{APPENDIX A: MICROSCOPIC DESCRIPTION}

In a microscopic description, the particle's trajectory in phase-space is known exactly. With this knowledge, we can construct a distribution function $f(x, p, Q)$ (without loss of generality, we shall consider only one particle):

$$
f(x, p, Q)=\int \frac{d \tau}{m} \delta^{(4)}(x-x(\tau)) \delta^{(4)}(p-p(\tau)) \delta^{\left(N^{2}-1\right)}(Q-Q(\tau))
$$


where $x(\tau), p(\tau)$ and $Q(\tau)$ obey the Wong equations (2.1), i.e. they naturally fulfill the mass-shell and Casimir constraints. For convenience, those constraints are here subsumed into the distribution function instead of being contained in the phase-space volume element. Had we not done this, (A1) would have to be written in terms of both a 3-dimensional $\delta$-function in momentum space, and an $N(N-1)$-dimensional $\delta$-function in color space.

We now prove that the expression (A1) for $f$ satisfies the collisionless Boltzmann equation (2.5). The first term in (2.5) can be rewritten, by using the properties of the $\delta$-function, as:

$$
p^{\mu} \frac{\partial}{\partial x^{\mu}} f=-\int \frac{d \tau}{m} p^{\mu}(\tau)\left[\frac{\partial}{\partial x^{\mu}(\tau)} \delta^{(4)}(x-x(\tau))\right] \delta^{(4)}(p-p(\tau)) \delta^{\left(N^{2}-1\right)}(Q-Q(\tau)) .
$$

From this, after using the Wong equation for the variable $x^{\mu}(\tau)$ and applying the chain rule, we get:

$$
p^{\mu} \frac{\partial}{\partial x^{\mu}} f=-\int d \tau\left[\frac{d}{d \tau} \delta^{(4)}(x-x(\tau))\right] \delta^{(4)}(p-p(\tau)) \delta^{\left(N^{2}-1\right)}(Q-Q(\tau)) .
$$

Similar arguments yield, for the second term in the Boltzmann equation:

$$
-g p^{\mu} Q_{a} F_{\mu \nu}^{a} \frac{\partial}{\partial p_{\nu}} f=-\int d \tau \delta^{(4)}(x-x(\tau))\left[\frac{d}{d \tau} \delta^{(4)}(p-p(\tau))\right] \delta^{\left(N^{2}-1\right)}(Q-Q(\tau))
$$

and for the third term:

$$
-g p^{\mu} f_{a b c} A_{\mu}^{b} Q^{c} \frac{\partial}{\partial Q_{a}} f=-\int d \tau \delta^{(4)}(x-x(\tau)) \delta^{(4)}(p-p(\tau))\left[\frac{d}{d \tau} \delta^{\left(N^{2}-1\right)}(Q-Q(\tau))\right] .
$$

Adding together the equations (A3), (A4) and (A5), we observe that the left hand side the $\tau$-integral of a total $\tau$-derivative - vanishes, thereby yielding the collisionless Boltzmann equation (2.5).

\section{APPENDIX B: CONSERVATION OF THE COLOR CURRENT}

Let us verify that the color current (2.8) is covariantly conserved. Using the collisionless transport equation, one can compute 


$$
\begin{aligned}
\partial_{\mu} j^{\mu a}(x) & =g \int d P d Q p^{\mu} Q^{a} \partial_{\mu} f(x, p, Q) \\
& =g^{2} \int d P d Q p^{\mu} Q^{a}\left(Q_{b} F_{\mu \nu}^{b}(x) \frac{\partial}{\partial p_{\nu}}+f^{d b c} A_{\mu b}(x) Q_{c} \frac{\partial}{\partial Q^{d}}\right) f(x, p, Q),
\end{aligned}
$$

where the color measure is $d Q=d^{\left(N^{2}-1\right)} Q C(Q)$, and $C(Q)$ specifies the color constraints in phase-space. Integrating by parts and discarding surface terms, one gets

$$
\begin{aligned}
\partial_{\mu} j^{\mu a}(x)= & -g^{2} \int d P\left[\int d Q\left(Q^{a} Q_{b} g_{\nu}^{\mu} F_{\mu \nu}^{b}(x)+p^{\mu} \delta_{c d} f^{d b c} A_{\mu b}(x) Q^{a}+p^{\mu} f^{d b c} A_{\mu b}(x) \delta_{d}^{a} Q_{c}\right)\right. \\
& \left.+\int d^{\left(N^{2}-1\right)} Q p^{\mu} A_{\mu b}(x) f^{d b c} Q_{c} \frac{\partial}{\partial Q^{d}} C(Q)\right] f(x, p, Q) .
\end{aligned}
$$

Among the four terms in the right side, only the third one survives. The first two terms cancel due to antisymmetry of $F_{\mu \nu}^{a}$ and $f^{d b c}$, respectively. The last term also cancels, since the constraints $C(Q)$ are gauge-invariant, i.e.,

$$
0=\delta Q^{a} \frac{\delta C(Q)}{\delta Q^{a}}=-g f^{a b c} \epsilon_{b}(x) Q_{c} \frac{\partial C(Q)}{\partial Q^{a}},
$$

where $\delta Q^{a}$ denotes an infinitesimal gauge transformation with arbitrary parameter $\epsilon_{b}(x)$. (For $S U(3)$ this last property can be explicitly checked by using the Jacobi-like identity $f_{a b c} d_{d e c}+f_{a d c} d_{e b c}+f_{a e c} d_{b d c}=0$.) Finally, one obtains the expression for the covariant conservation of the color current: $\partial_{\mu} j^{\mu a}(x)+g f^{a b c} A_{\mu b}(x) j_{c}^{\mu}(x)=0$. 


\section{REFERENCES}

[1] P.F. Kelly, Q. Liu, C. Lucchesi and C. Manuel, Phys. Rev. Lett. 72, 3461 (1994).

[2] See e.g. E. Braaten and R. Pisarski, Nucl. Phys. B337, 569 (1990), reference [3].

[3] R. Pisarski, Phys. Rev. Lett. 63, 1129 (1989).

[4] E. Braaten and R. Pisarski, Phys. Rev. D 42, 2156 (1990); 45, 1827 (1992); Nucl. Phys. B337, 569 (1990); B339, 310 (1992).

[5] J. Frenkel and J.C. Taylor, Nucl. Phys. B334, 199 (1990).

[6] J.C. Taylor and S. Wong, Nucl. Phys. B346, 115 (1990).

[7] R. Efraty and V.P. Nair, Phys. Rev. Lett. 68, 2891 (1992); Phys. Rev. D 47, 5601 (1993).

[8] R. Jackiw and V.P. Nair, Phys. Rev. D 48, 4991 (1993).

[9] J.-P. Blaizot and E. Iancu, Phys. Rev. Lett. 70, 3376 (1993); Nucl. Phys. B417, 608 (1994).

[10] J.M. Cornwall, R. Jackiw and E. Tomboulis, Phys. Rev. D 10, 2428 (1974).

[11] R. Jackiw, Q. Liu and C. Lucchesi, MIT preprint CTP \# 2261, November 1993, to appear in Phys. Rev. D.

[12] E. Lifshitz and L. Pitaevskii, Physical Kinetics (Landau and Lifshitz Course of Theoretical Physics, Volume 10), Pergamon Press, Oxford, 1981.

[13] V.P. Silin, Zh. Eksp. Teor. Fiz. 38, 1577 (1960) [Engl. transl.: Sov. Phys. JETP 11, 1136 (1960)].

[14] U. Heinz, Phys. Rev. Lett. 51, 351 (1983); Ann. Phys. (NY) 161, 48 (1985); Ann. Phys. (NY) 168, 148 (1986);

H.-Th. Elze and U. Heinz, Phys. Rep. 183, 81 (1989). 
[15] V. Klimov, Sov. J. Nucl. Phys. 33, 934 (1981);

H.A. Weldon, Phys. Rev. D 26, 1394 (1982).

[16] S. Wong, Nuovo Cim. 65A, 689 (1970).

[17] J.-P. Blaizot and E. Iancu, Phys. Lett. B326, 138 (1994).

[18] K. Johnson, Ann. Phys. (N.Y.) 192, 104 (1989).

[19] A. Alekseev, L. Faddeev and S. Shatashvili, J. Geom. Phys. 3, 1 (1989). 